\title{
Bioprosthetic valve thrombosis: The harder one looks, the more one finds
}

\author{
Michael Mack, MD, ${ }^{\mathrm{a}}$ and David Holmes, $\mathrm{MD}^{\mathrm{b}}$
}

From the ${ }^{\mathrm{a}}$ Heart Hospital, Baylor Plano, Plano, Tex; and the ${ }^{\mathrm{b}}$ Mayo Clinic, Rochester, Minn.

Disclosures: Authors have nothing to disclose with regard to commercial support.

Received for publication July 9, 2016; accepted for publication July 12, 2016; available ahead of print Aug 12, 2016.

Address for reprints: Michael Mack, MD, Heart Hospital, Baylor Plano, 1100 Allied Dr, Plano, TX 75093

(E-mail: Michael.mack@bswhealth.org).

J Thorac Cardiovasc Surg 2016;152:952-3

0022-5223/\$36.00

Copyright (c) 2016 by The American Association for Thoracic Surgery

http://dx.doi.org/10.1016/j.jtcvs.2016.07.024

The desire to avoid anticoagulation after surgical aortic valve replacement (SAVR) has been one of the major drivers toward the greater use of bioprosthetic valves and less use of mechanical valves so that more than $90 \%$ of surgical valves now implanted are tissue. Recently concerns have been raised about the occurrence of bioprosthetic valve thrombosis (BPVT) and its possible relationship to subsequent structural valve deterioration. ${ }^{1}$ Recent studies done with sophisticated imaging techniques, including 4-dimensional computed tomography (4D CT) with highspeed scanners have clearly documented leaflet immobility, thickness, and thrombosis both early and late after both SAVR and transcatheter aortic valve replacement (TAVR) procedures (Figure 1). ${ }^{2}$ Although the true incidence and clinical relevance of these different findings are as yet unknown, it is clear that the phenomenon is more common than was previously appreciated.

As yet there have not been surveillance studies performed to answer definitively the question of the true incidences of BPVT, leaflet immobility, and valvular hemodynamic deterioration; however, here is what we do know from published series. First, when the presence of a change in mean transvalvular gradient on serial transthoracic echocardiography was used as the definition of valvular hemodynamic deterioration, an incidence in TAVR of $4.5 \%$ was found usually within 30 days of TAVR implantation. ${ }^{4}$ Other series have documented improvement in gradients with anticoagulation leading to the presumption of leaflet thrombosis as the cause at least in some patients. Various predisposing factors for BPVT appear to be small valve size, high body mass index, lack of anticoagulation, and a TAVR valve in a previously placed surgical valve.

So what do we now know about BPVT? First, it is more common than previously appreciated. Second, 4D CT is the most sensitive imaging modality for detection (Figure 1). Third, anticoagulation appears to resolve the problem, at least in a subset of patients.

Yet there is an even greater amount of information we do not know, including the following:

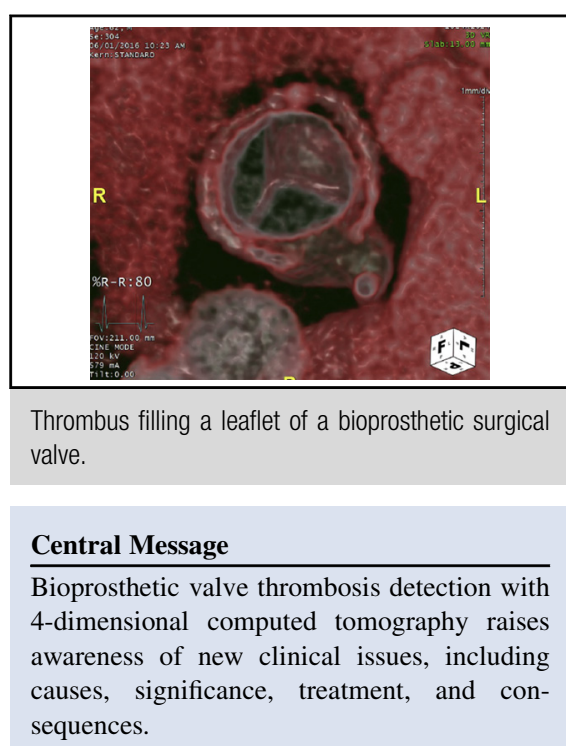

See Editorial page 975.

1. What is the true incidence of valve leaflet thrombosis?

2. Is it more common in TAVR or in SAVR?

3. Is it more common with porcine or bovine pericardial valves?

4. Is it more common with balloon-expandable or selfexpanding TAVR valves?

5. What are the predisposing factors?

6. When is it most likely to occur? Early? Late? Does the temporal profile of the abnormality vary with TAVR and SAVR and the type of valve?

7. Is periprocedural adjunctive therapy protective? If so, what is the optimal strategy, dual antiplatelet therapy, vitamin $\mathrm{K}$ antagonists, or novel oral anticoagulants? What is the appropriate duration of therapy?

8. What is the clinical relevance of leaflet thrombosis found on imaging studies?

9. Does leaflet thrombosis explain the pathophysiology of subsequent premature structural valve deterioration?

To answer these questions, the US Food and Drug Administration as part of investigational device exemption trials of TAVR versus SAVR in patients with low surgical risk has mandated that surveillance substudies with $4 \mathrm{D}$ CT for BPVT be performed. A total of 800 patients (400 TAVR and 400 SAVR) will be studied in 2 trials. It is to be hoped that these studies will shed further light on this issue. 


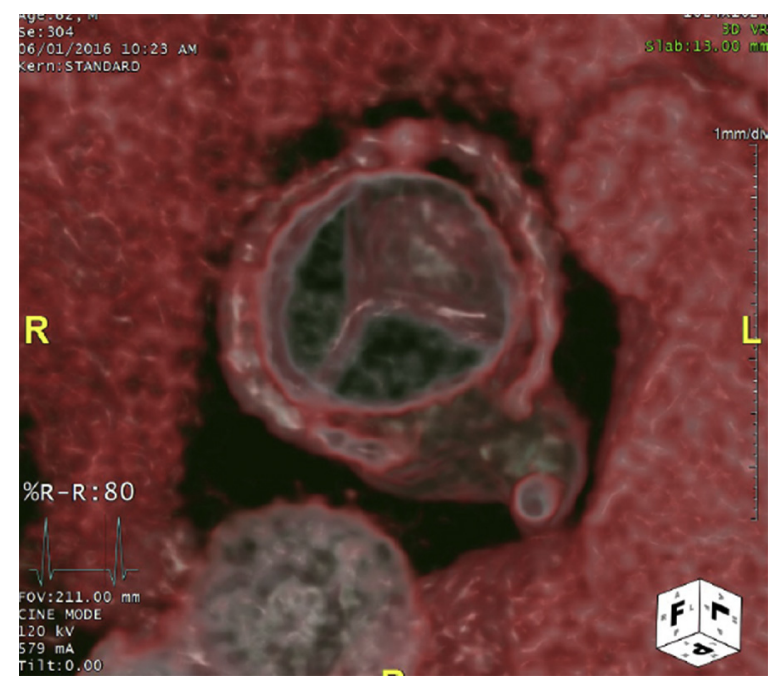

FIGURE 1. Thrombus filling a leaflet of a bioprosthetic surgical valve 2.5 years after implantation.

Pending these results, what should be current clinical practice? Postprocedural anticoagulation with warfarin is currently a IIb indication in the 2014 American College of Cardiology and American Heart Association Valvular Guidelines for 3 months in patients undergoing SAVR. Unless there are contraindications, consideration of anticoagulation with warfarin should be given to both patients undergoing SAVR and those undergoing TAVR. Routine surveillance after both TAVR and SAVR with transthoracic echocardiography should be performed initially at 30 days and then yearly. If a change in mean gradient greater than $10 \mathrm{~mm} \mathrm{Hg}$ or a clinical event (including stroke, persistent or recurrent heart failure, decrease in ejection fraction, or new paravalvular leak) occurs, 4D CT (alternatively transesophageal echocardiography if 4D CT is not feasible) should be performed. If abnormal findings are detected, a trial of anticoagulation should be considered. If resolution does not occur, consideration should be given to thrombolysis or surgical intervention, as dictated by the clinical situation.

The magnitude of the issue and the appropriate clinical management represent significant current unknowns. One thing is clear, however; the harder one looks, the more one finds. Clinicians should be vigilant in detecting and treating BPVT. Ongoing studies should bring the significance, clinical relevance, and optimal management of these findings into a clearer focus.

\section{References}

1. Egbe AC, Pislaru SV, Pellikka PA, Poterucha JT, Schaff HV, Maleszewski JJ, et al Bioprosthetic valve thrombosis versus structural failure: clinical and echocardiographic predictors. J Am Coll Cardiol. 2015;66:2285-94.

2. Makkar RR, Fontana G, Jilaihawi H, Chakravarty T, Kofoed KF, de Backer O, et al. Possible subclinical leaflet thrombosis in bioprosthetic aortic valves. $N$ Engl J Med. 2015;373:2015-24.

3. Egbe AC, Connolly HM, Schaff HV. Bioprosthetic valve thrombosis: what we know and what we need to know. J Thorac Cardiovasc Surg. 2016;152:975-8.

4. Del Trigo M, Muñoz-Garcia AJ, Wijeysundera HC, Nombela-Franco L, Cheema AN, Gutierrez E, et al. Incidence, timing, and predictors of valve hemodynamic deterioration after transcatheter aortic valve replacement: multicenter registry. J Am Coll Cardiol. 2016;67:644-55. 